\title{
Altered gut microbiota due to daily intake of flavonoid- rich orange juice regulate depressive symptoms
}

\author{
Miey Park \\ Gachon University \\ Jihee Choi \\ Gachon University \\ Hae-Jeung Lee ( $\nabla$ skysea1010@gmail.com ) \\ Gachon University https://orcid.org/0000-0002-9124-0004
}

\section{Research}

Keywords: Depression, Microbiota, Flavonoid treatment, Lachnospiraceae

Posted Date: April 1st, 2020

DOI: https://doi.org/10.21203/rs.3.rs-19114/v1

License: (ㅇ) (1) This work is licensed under a Creative Commons Attribution 4.0 International License. Read Full License 


\section{Abstract}

Background: Depression is not just a general mental health problem but a serious medical illness that can worsen without treatment. The gut microbiome plays a major role in the two-way communication system between the intestines and brain. The current study examined the effects of flavonoids on depression by observing the changes in the gut microbiome and depressive symptoms of young participants consuming flavonoid-rich orange juice.

Results: All participants (Depression) were evaluated using the Center for Epidemiological Studies Depression Scale (CES-D), a psychiatric screening tool used to detect preexisting psychiatric disorders, and were randomly divided into two groups: the orange juice (Depression_0) and flavored placebo (Depression_F) groups. For 8 weeks, flavonoid-rich orange juice $(157.9 \pm 1.4 \mathrm{mg} / 100 \mathrm{~g})$ or flavored placebo drink $(28.4 \pm 0.7 \mathrm{mg} / 100 \mathrm{~g})$ were consumed twice daily before breakfast and dinner. In total, 80 fecal samples from 40 participants (mean age, 21.83 years) were sequenced. Regarding depression, positive correlations were observed between brain-derived neurotrophic factor and the Lachnospiraceae family (Lachnospiraceae_uc and Murimonas) before flavonoidrich orange juice treatment. Most notably, the abundance of the Lachnospiraceae family (Lachnospiraceae_uc, Eubacterium_g4, Roseburia_uc, Coprococcus_g2_uc, Agathobacter_uc) had increased after flavonoid-rich orange juice treatment compared to that after flavored placebo treatment. We also validated the presence of unclassified Lachnospiraceae through sensitive real-time quantitative polymerase chain reaction using stool samples from participants before and after flavonoid treatment.

Conclusions: Our results provide novel interventional evidence that alteration in the microbiome due to flavonoid treatment is related to potential improvement in depression in young adults.

Trial registration: This study was registered on the Clinical Research Information Service (CRIS) with the registration number КСT0004865.

\section{Background}

Depression is a common psychiatric disorder and is accompanied by symptoms such as sadness, decrease in motivation or interest, low self-esteem, sleep deprivation, loss of appetite, fatigue, and loss of concentration [1]. Moreover, depression can lead to serious problems in daily or social life and may also result in suicide. It is being increasingly recognized that few clinical symptoms are found in healthy populations [2]. It is estimated that the number of individuals with depression worldwide is 350 million, and the population affected by depression is gradually expanding $[1,3]$.

Dietary patterns and depression are closely related [4, 5]. Previous studies have reported that the intake of sweets, red and processed meat, high-fat dairy products, refined grains and low intake of fruits and vegetables may increase the risk of depression [5-7]. Particularly, flavonoids and polyphenols (micronutrients) are abundant in fruits, vegetables, tea, and cocoa. Previous studies have revealed that flavonoid intake increases the amount of blood delivered to the brain, thereby improving brain cognitive function [8-11]. Epidemiological studies $[12,13]$ and clinical studies $[14,15]$ have also reported that high flavonoid intake through vegetables and fruits helps lower the risk of depression. Although fruits and fruit juices can easily supply flavonoids, such 
as hesperidin and narirutin, studies on their effectiveness on the human body are still at an early stage, and further studies are needed.

There have been increasing number of observational studies on the associations between habitual quality of diet and the prevalence of depression [16]. Additionally, it has been reported that dietary flavonoids (polyphenols) are associated with gut microbiome regulation, and these microbiomes can prevent and treat depression by increasing the synthesis of serotonin, which is a neurotransmitter in the body [16-18]. An experimental animal model showed that probiotics may reduce pro-inflammatory immune responses and increase levels of the serotonergic precursor, tryptophan, thereby ameliorating depressive symptoms [19]. Furthermore, depression can be reversed by administering the probiotic Bifidobacterium infantis to male Sprague-Dawley rats $(n=20)[20]$.

Tryptophan, as a precursor, is an essential amino acid needed by the body to synthesize serotonin [18]. As an essential amino acid, tryptophan is not synthesized from simple substances in humans and other animals; thus, it must be obtained through protein-based foods and dietary proteins. Microbes and plants generally synthesize tryptophan from shikimic acid or anthranilate, and human gastrointestinal microbiota metabolize tryptophan into indole and subsequently 3-indolepropionic acid, a powerful neuroprotective antioxidant that scavenges free radicals [21-23].

Probiotics, including Bifidobacteria and Lactobacilli, also alleviate the immune response, decrease pathogens, and maintain the intestinal microbiota in subjects receiving antibiotic treatment [24-26]. It plays a major role in two-way communication between the intestines and brain $[26,27]$. Moreover, the impact of probiotics on human psychiatric disorders has recently emerged as an area of interest in neuroscience [28].

The activity of microbial metabolites is mainly due to their ability to permeate the blood-brain barrier. Dietary polyphenols are metabolized by microorganism in the colon, and microbial metabolites of polyphenol, isoflavones, and lignans generally show greater permeability through the man-made gut and blood-brain barrier than their parent compound [29]. Another polyphenol metabolite, gallic acid derivatives, demonstrated neuroprotective effects through the modulation of the nuclear factor KB (NF-kB) pathway [30]. Polyphenols may affect the composition of the gut microbiota, and bioactive compounds in polyphenol metabolites produce clinical benefits [31].

Although previous studies have indicated that flavonoids consumed through the diet could affect gut microbiome regulation and depression, there have been limited intervention studies on the microbial metabolites of polyphenol. Therefore, this study aimed to evaluate the effects of flavonoids on depression in young adults by observing the changes in the gut microbiome and depressive symptoms of participants consuming flavonoid-rich orange juice. Furthermore, this study attempted to establish a theoretical basis for providing basic data and policy direction to develop a dietary program for alleviating and preventing depressive symptoms.

\section{Results}

\section{Characteristics of the study participants}


The study participants (40) consisted of 16 men ( $40 \%)$ and 24 women (60\%). The mean age was $21.83 \pm 2.43$ years, and there was no dropout. Statistical analysis was conducted for participant characteristics. Baseline characteristics did not differ between participants in the flavonoid-rich orange juice (Orange) group and lowflavonoid drink (Flavored) groups (Supplement Table 1).

Table 1

Anthropometric data and blood test results at baseline and 8 weeks after intervention.

\begin{tabular}{|c|c|c|c|c|c|c|c|}
\hline \multirow[t]{3}{*}{ Variables } & \multicolumn{2}{|c|}{ Orange $(n=20)$} & \multirow{3}{*}{$\begin{array}{l}\text { p- } \\
\text { value }^{\dagger}\end{array}$} & \multicolumn{2}{|c|}{ Flavored $(n=20)$} & \multirow[t]{3}{*}{ p-value ${ }^{\dagger}$} & \multirow{3}{*}{$\begin{array}{l}\Delta \text { group } \\
\text { comparison }^{*}\end{array}$} \\
\hline & Baseline & $\begin{array}{l}\text { After } \\
\text { intervention }\end{array}$ & & Baseline & $\begin{array}{l}\text { After } \\
\text { intervention }\end{array}$ & & \\
\hline & \multicolumn{2}{|c|}{ Mean \pm SE } & & \multicolumn{2}{|c|}{ Mean \pm SE } & & \\
\hline Weight, kg & $\begin{array}{l}66.28 \pm \\
3.41\end{array}$ & $\begin{array}{l}66.57 \pm \\
3.40\end{array}$ & $0.382^{\dagger}$ & $\begin{array}{l}60.22 \pm \\
2.32\end{array}$ & $\begin{array}{l}59.98 \pm \\
2.31\end{array}$ & $0.510^{\dagger}$ & 0.672 \\
\hline $\mathrm{BMI}, \mathrm{kg} / \mathrm{m}^{2}$ & $\begin{array}{l}23.45 \pm \\
0.87\end{array}$ & $\begin{array}{l}23.62 \pm \\
0.88\end{array}$ & $0.178^{\dagger}$ & $\begin{array}{l}21.74 \pm \\
0.66\end{array}$ & $\begin{array}{l}21.62 \pm \\
0.63\end{array}$ & $0.387^{\dagger}$ & 0.122 \\
\hline $\begin{array}{l}\text { Percent body } \\
\text { fat, \% }\end{array}$ & $\begin{array}{l}27.72 \pm \\
1.76\end{array}$ & $\begin{array}{l}27.90 \pm \\
1.85\end{array}$ & $0.609^{\dagger}$ & $\begin{array}{l}25.84 \pm \\
2.08\end{array}$ & $\begin{array}{l}24.73 \pm \\
2.18\end{array}$ & $0.052^{\dagger}$ & 0.050 \\
\hline SBP, mmHg & $\begin{array}{l}121.25 \\
\pm 2.98\end{array}$ & $\begin{array}{l}123.05 \pm \\
2.78\end{array}$ & $0.520^{\dagger}$ & $\begin{array}{l}121.20 \\
\pm 2.39\end{array}$ & $\begin{array}{l}118.40 \pm \\
3.55\end{array}$ & $0.307^{\dagger}$ & 0.117 \\
\hline DBP, mmHg & $\begin{array}{l}74.80 \pm \\
2.01\end{array}$ & $\begin{array}{l}76.20 \pm \\
1.63\end{array}$ & $0.522^{\dagger}$ & $\begin{array}{l}70.55 \pm \\
2.24\end{array}$ & $\begin{array}{l}72.60 \pm \\
1.47\end{array}$ & $0.397^{+}$ & 0.063 \\
\hline BDNF & $\begin{array}{l}255.30 \\
\pm 40.78\end{array}$ & $\begin{array}{l}322.08 \pm \\
42.80\end{array}$ & $0.251^{\dagger}$ & $\begin{array}{l}267.23 \\
\pm 45.00\end{array}$ & $\begin{array}{l}287.45 \pm \\
53.24\end{array}$ & $0.673^{\dagger}$ & 0.132 \\
\hline $\begin{array}{l}\text { Serotonin, } \\
\mathrm{ng} / \mathrm{mL}\end{array}$ & $\begin{array}{l}151.73 \\
\pm 22.76\end{array}$ & $\begin{array}{l}187.66 \pm \\
27.12\end{array}$ & $0.219^{\dagger}$ & $\begin{array}{l}122.62 \\
\pm 13.37\end{array}$ & $\begin{array}{l}154.23 \pm \\
20.69\end{array}$ & $0.102^{\dagger}$ & 0.058 \\
\hline Folate, ng/mL & $\begin{array}{l}6.31 \pm \\
0.69\end{array}$ & $7.47 \pm 1.00$ & $0.013^{\dagger}$ & $\begin{array}{l}6.39 \pm \\
1.45\end{array}$ & $6.72 \pm 3.41$ & $0.536^{\dagger}$ & 0.057 \\
\hline $\begin{array}{l}\text { Homocysteine, } \\
\mu \mathrm{mol} / \mathrm{L}\end{array}$ & $\begin{array}{l}10.66 \pm \\
3.10\end{array}$ & $\begin{array}{l}13.51 \pm \\
4.48\end{array}$ & $0.001^{\dagger}$ & $\begin{array}{l}11.55 \pm \\
6.47\end{array}$ & $\begin{array}{l}19.47 \pm \\
15.27\end{array}$ & $0.001^{\ddagger}$ & 0.007 \\
\hline hs-CRP, mg/L & $\begin{array}{l}1.76 \pm \\
0.56\end{array}$ & $0.81 \pm 0.29$ & $0.180^{\ddagger}$ & $\begin{array}{l}2.03 \pm \\
0.89\end{array}$ & $0.41 \pm 0.10$ & $0.061^{\ddagger}$ & 0.031 \\
\hline $\begin{array}{l}\text { Vitamin } B_{12} \\
\mathrm{pg} / \mathrm{mL}\end{array}$ & $\begin{array}{l}517.70 \\
\pm 30.57\end{array}$ & $\begin{array}{l}507.75 \pm \\
25.80\end{array}$ & $0.694^{\dagger}$ & $\begin{array}{l}550.45 \\
\pm 45.51\end{array}$ & $\begin{array}{l}542.00 \pm \\
38.89\end{array}$ & $0.768^{\dagger}$ & 0.143 \\
\hline CES-D score & $\begin{array}{l}30.4 \pm \\
7.97\end{array}$ & $\begin{array}{l}15.15 \pm \\
8.95\end{array}$ & $\begin{array}{l}< \\
0.0001^{\dagger}\end{array}$ & $\begin{array}{l}28.75 \pm \\
7.26\end{array}$ & $\begin{array}{l}15.15 \pm \\
8.95\end{array}$ & $<.<0001 \dagger$ & 0.889 \\
\hline
\end{tabular}




\section{Statistical analysis of the food frequency questionnaire and 24- $h$ recall results}

Before conducting this study, the validated semiquantitative food frequency questionnaire (FFQ) was used to understand the eating habits of the study participants in the past 1 year [32]. At the end of the study, we evaluated the participants for the same items of the FFQ as those used at baseline to check if the dietary intake pattern had changed in 2 months. Each food was assessed in terms of the intake frequency per person per day and then compared between the groups. The results of the FFQ analysis revealed that the intake of all nutrients decreased from the baseline to the end of the treatment in the Orange group. Particularly, the intake of energy, protein, fat, fiber, calcium, phosphate, iron, sodium, potassium, vitamin A, $\beta$-carotene, riboflavin, and niacin decreased significantly $(p<0.05)$. In the Flavored group, there were no changes in the intake of energy, phosphate, thiamin, and niacin. However, the intake of carbohydrate increased significantly $(p<0.05)$, and the intake of other nutrients decreased significantly $(p<0.05)$ (Supplement Table 2).

Table 2

Oligonucleotides used for quantitative real-time polymerase chain reaction assays.

\begin{tabular}{|lllll|}
\hline Species & Primer sequences & & Size & Reference \\
\cline { 2 - 5 } & Forward & Reverse & & \\
\hline Lachnospiraceae_uc & AACAGAGGAGACAGGTGGTG & GCTTCCCTTTGTTTACGCCA & 228 & This study \\
\hline Roseburia_uc & CGCAGCAAACGCAATAAGTA & ACCACTGCTCCGAAGAGAAA & 205 & This study \\
\hline Bifidobacterium_uc & TCGGGGTGAGTGTACCTTTC & AATTCCAGTCTCCCCTACCG & 184 & This study \\
\hline Universal primers & CGGTGAATACGTTCYCGG & AAGGAGGTGATCCRGCCGCA & 172 & $\begin{array}{l}\text { Suzuki et } \\
\text { al. 2000 }\end{array}$ \\
\hline
\end{tabular}

The results of the 24-h recall showed that in the Orange group, only the intake of energy, protein, fat, phosphate, sodium, and vitamin B12 decreased significantly $(p<0.05)$, and that of folate and vitamin $C$ increased significantly $(p<0.05)$. In the Flavored group, the intake of riboflavin and niacin significantly decreased $(p<$ 0.05 ), while that of other nutrients did not change. The intake of folate and vitamin $C$ intake was significantly ( $p$ $<0.05$ ) different between the Orange and Flavored groups (Supplement Table 3).

\section{Comparison of hematological profiles and anthropometric measurements}

Blood test results revealed an increase in brain-derived neurotrophic factor (BDNF) levels in the Orange group (Table 1). The numerical increase in the levels in the Orange group was larger than that in the Flavored group but was not significantly different $(p<0.05)$. The serotonin level in the Orange group was high but was not significantly higher than that in the Flavored group, and there was no significant difference between the two groups $(p=0.058)$. Folate and homocysteine levels showed significant differences at baseline and postintervention in the Orange group $(p<0.05)$. Homocysteine intake in the Flavored group was significantly different and fell out of the normal range (5.0-15.0 $\mu \mathrm{mol} / \mathrm{L})$. Moreover, after adjusting for sex, age, household 
income, and education level, body fat (\%), homocysteine level, and high-sensitivity C-reactive protein (hs-CRP) level were significantly $(\mathrm{p}<0.05)$ different between the Orange and Flavored groups (Table 1).

\section{Comparison of the Center for Epidemiological Studies Depression Scale scores}

Post-intervention, the mean Center for Epidemiological Studies Depression Scale (CES-D) scores in the Orange and Flavored groups decreased to $<20$ points (Table 1). Moreover, adjusted (sex, age, education, and household income) multiple regression analysis showed that the $\mathrm{p}$-values of the CES-D score in both the Orange and Flavored groups decreased significantly after the intervention $(p<0.0001)$.

\section{Sequencing characteristics and changes in microbial diversity in patients with depression}

In total, 80 fecal samples from 40 participants before and after the intervention were sequenced on the Illumina MiSeq sequencer. The sequencing results were obtained from 40 untreated participants (CES-D score $\geq 21$ ), who were randomly divided into the Orange group (20 participants) and Flavored group (20 participants) for the analysis.

Phylogenetic alpha-diversity indexes (Chao, Phylogenetic Diversity, Shannon, and Simpson) were used to assess gut microbial diversity in participants with depression before and after flavonoid treatment (Fig. 1). Gut microbial diversity, as estimated by Chao, was greater in the Depression group than in the Orange $(p=0.371)$ and Flavored ( $p=0.090)$ groups (Fig. 1A). Moreover, the Depression group plot had significantly higher phylogenetic diversity than the Orange $(p=0.019)$ and Flavored $(p=0.002)$ group plots (Fig. 1B). However, diversity as measured using the Shannon and Simpson index had no significant differences between the Depression and Orange groups (Fig. 1C-D).

\section{Changes in microbiota taxonomic composition in the Depression_0 and Orange groups}

Comparisons based on taxonomy were performed to determine the differences between the microbiota of 20 individuals with depression (before orange treatment group [Depression_O]) and 20 participants in the Orange group. At the phylum level, Firmicutes were more abundant in the gut in the Depression_0 group than in the Orange group. Compared with the Depresssion_0 group, the Bacteroidetes and Actinobacteria phyla were increasingly abundant in the Orange group. There was higher abundance of the Ruminococcaceae and Erysipelotrichaceae families in the Depression_0 group, while the Lachnospiraceae, Bifidobacteriaceae, and Akkermansiaceae families were more abundant in the Orange group. Several differences between the Depression_O and Orange groups were observed at the genus level. Lactobacillus, Alistipes, Roseburia, unclassified Lachnospiraceae (Lachnospiraceae_uc), Akkermansia, Bifidobacterium, and Collinsella abundance rates were higher, while Faecalibacterium, Streptococcus, and Eubacterium_g23 abundance rates were lower in the Orange group (Fig. 2A). Further, we also used metagenome analysis to validate the linear discriminant analysis (LDA) effect size (LEfSe) and found that eight families (Bifidobacteriaceae, Akkermansiaceae, Bacteroidaceae, Veillonellaceae, Coriobacteriaceae, Lachnospiraceae, Lactobacillaceae, and Rikenellaceae) were abundant in the Orange group (Fig. 2A). An LDA analysis $(p<0.05$, LDA score $>2)$ showed that ten taxons were more abundant in the Orange group (Fig. 2B). Lachnospiraceae (Roseburia, Coprococcus_g2, 
Agathobacter, Clostridium_g24, and Eubacterium_g4), Bifidobacteriaceae (Bifidobacterium), and Bacteroidaceae (Bacteroides), which were enriched in the Orange group, were the major phylotypes that contributed to the difference in the gut microbiota composition between the Depression_0 and Orange groups.

\section{Changes in microbiota taxonomic composition in the Depression_F and Flavored groups}

The gut microbiota composition of 20 individuals with depression (before flavored treatment group [Depression_F]) and 20 participants in the Flavored groups showed differences in two phyla, Firmicutes and Bacteroidetes. The relative abundance of Firmicutes was greater in the Flavored group than in the Depression_F group, while Bacteroidetes had a lower abundance rate in this group (Fig. 3A). At the family level, Ruminococcaceae, Bacteroidaceae, and Bifidobacteriaceae were less abundant in the Flavored group, but Lachnospiraceae, Coriobacteriaceae, Streptococcaceae, and Lactobacillaceae were more abundant in this group. Bacteroides and Bifidobacterium were enriched in the Depression_F group compared with that in the Flavored group at the genus level (Fig. 3A). The LEfSe analysis also showed that the Bacteroidetes phylum and Bacteroides and Subdoligranulum genera were enriched in the Depression_F group, while the Firmicutes phylum and Blautia, Streptococcus, and Coprococcus genera were abundant in the Flavored group (Fig. 3A). LDA analysis ( $p<0.05$, LDA score $>2$ ) revealed that Lachnospiraceae (Blautia_uc and Bifidobacterium_uc) were more abundant in the Flavored group than in the Depression_F group (Fig. 3B).

\section{Association between gut microbiota and depression}

We also evaluated the associations of serum biomarkers with the relative abundances of gut microbiota. We found that depression and serum biomarkers were closely associated with gut microbiota in the Depression group (Fig. 4). The serum BDNF level was significantly positively correlated with the abundance of the Lachnospiraceae family (Lachnospiraceae_uc and Murimonas) and was negatively correlated with OCTT_g (Ruminococcaceae) abundance in those with depression. The relative abundances of Fusicatenibacter, PAC001043_g, and Eubacterium_g20 were positively correlated with body mass index (BMI), while those of the Ruminococcaceae family (PAC001100_g and OCTT_g) and Christensenella genus were negatively correlated with BMI $(p<0.001)$. Additionally, homocysteine levels were positively correlated with the abundance of the Gemella genus $(p<0.01)$ in patients with depression.

\section{Flavonoid-rich drink increases the relative abundance of Lachnospiraceae_uc and Bifidobacterium_uc in patients with depression}

To explore the contribution of the flavonoid-rich orange drink to the observed changes in gut microbial compositions in those with depression, we conducted a real-time polymerase chain reaction (PCR) experiment with stool samples from the Depression group and Orange and Flavored groups. We used the universal bacterial gene for copy control and another normal stool sample (cohort) for internal control (Table 2). The results of realtime PCR showed that the relative abundance of Lachnospiraceae_uc and Bifidobacterium_uc was significantly increased in the Orange group compared to that in the Depression_0 group (Fig. 5A and C). However, the relative expression of Lachnospiraceae_uc was increased and that of Bifidobacterium_uc was decreased in the Flavored 
group compared with those in the Depression_F group, but there was no significant difference (Fig. 5D and F). The relative expression of Roseburia_uc in the Orange group was increased compared with that in the Flavored group, but no significant difference was noted (Fig. 5B and E).

\section{Discussion}

This intervention study characterized the gut microbiota in the Depression (CES-D scores $\geq 21$, untreated) group compared with those in the flavonoid-rich versus placebo treatment groups. Furthermore, the associations between gut microbiota and depression were investigated. In this study, the 8-week flavonoid intervention changed the gut microbiota taxonomic composition and diversity in the Depression group. The gut microbiota composition in the Depression group was dominantly characterized by Firmicutes. However, a clinical report on major depressive disorder (MDD) has shown that low counts of Firmicutes may lead to a reduction in shortchain fatty acids, which can serve as a physiological basis for low levels of inflammation against depression [33]. However, no consensus results have emerged from clinical studies on depression and gut microbiota taxonomic composition that are relevant to depression [34].

We found that the generally dominant bacteria in the Orange group were unclassified Lachnospiraceae, unclassified Bacteroides, Eubacterium_g4, Clostridium_g24, unclassified Bifidobacterium, Eubacterium ventriosum, unclassified Roseburia, Coprococcus_g2, and unclassified Agathobacter. The genera that most significantly increased in number in the Orange group were unclassified Lachnospiraceae, unclassified Roseburia, and unclassified Bifidobacterium $(p<0.001)$. Intestinal microbial groups are affected by environmental factors, such as food and the presence of parasites, and genetic factors may also cause such differences [35]. Despite the vast diversity of gut bacteria, only 13 identified core bacterial genera, including unclassified Lachnospiraceae, were common among $95 \%$ of individuals [36].

Naseribafrouei et al. (2014) reported that the abundance of higher-order Bacteroidales and the Oscillibacter and Alistipes genera and lower abundance of the family Lachnospiraceae were associated with depression in the comparison of the gut microbiota between 37 patients with depression and 18 healthy controls [37]. Moreover, Jiang et al. (2015) reported that the abundance of Lachnospiraceae and Ruminococcaceae decreased in patients with MDD compared with that in the control group [38]. In our study, after the intervention, the relative taxonomic abundance of Lachnospiraceae increased in both groups (Orange and Flavored) compared with that in the Depression group. Furthermore, the abundance of genus Lachnospiraceae significantly increased $(p<$ $0.0001)$ in the Orange group compared with that in the Flavored group $(p=0.0002)$ (Supplementary Fig. 1A and 2A). Moreover, significantly increased expression of Lachnospiraceae_uc and Roseburia_uc were observed in the Orange group compared with that in the Flavored group as shown by real-time PCR results of stool samples (Fig. 5). Thus, we found that Lachnospiraceae responds to flavonoid treatment in patients with depression.

In the Orange group, the relative taxonomic abundance of Bifidobacterium, Roseburia, Ruminococcus, and Akkermansia significantly increased compared to that in the Flavored group (Supplement Figs. 1 and 2). The relative expression of Bifidobacterium_uc in the Orange group was also significantly increased compared to that in the Flavored group (Fig. 5C and F). Bifidobacterium is a genus that influences intestinal function in infants who have received healthy breast milk, while low but relatively stable Bifidobacterium counts are observed in adulthood [39]. The abundance of Bifidobacterium increases in the late stages of pregnancy in both women and mice, indicating the causative role of progesterone [40]. Bifidobacterium bifidum colonization increases 
interleukin 6 (IL-6) and IL-8 cytokine levels through NF-KB activation in mice [41]. Supplementation of Bifidobacterium has also been shown to increase the fecal levels of immunoglobulin $A$ in young women [42] and to lead to changes in the levels of human immune cells [43]. Furthermore, exogenous probiotic microbes, such as Bifidobacterium and Lactobacillus, have been shown to reduce anxiety in human participants [44].

elative abundances of bacterial genera, including Akkermansia spp., were significantly reduced in socially defeated animals, which was positively correlated with both anxiety and depression [45]. Moreover, the administration of prebiotics increased the relative abundance of Akkermansia during exposure to stressors [46, 47]. The abundance of Akkermansia has been suggested as a biomarker for healthy intestines and has an inverse correlation with several intestinal disorders [48]. Generally, Akkermansia and Bifidobacterium are wellknown health-associated genera that protect against inflammation, promote immunomodulation, and promote healthy metabolic homeostasis [49]. These findings corroborate our study on flavonoid intervention, and the relative abundance of Akkermansia significantly increased in the Orange group compared to that in the Flavored group.

Greater microbial diversity was found in the Depression group than that in the Orange and Flavored groups. To date, four studies on MDD have investigated and performed a microbial diversity analysis. While three studies reported no significant differences in microbial diversity [37, 50, 51], Jiang et al. (2015) reported greater microbial diversity in patients with MDD than in healthy individuals [38]. High microbial diversity could be easily affected by age, eating habits, and other factors [52]. Although greater diversity of bacteria is potentially beneficial to human health, the precise consequences of increased bacterial diversity for depression are still unclear [38].

In our study, the abundances of Ruminococcaceae and Lachnospiraceae were positively correlated with BDNF levels in the Depression group. After the 8-week intervention, the serum levels of BDNF in the Orange group were higher than those in the Flavored group. The abundances of Ruminococcaceae OCTT_g $(p<0.0016)$, Ruminococcus_g2 ( $p<0.007)$, Ruminicoccus $(p<0.0248)$, and Lachnospiraceae_uc $(p<0.039)$ were positively correlated with serum BDNF levels. BDNF is a neurotrophin that performs multiple functions in the central nervous system and participates in the therapeutic mechanisms of antidepressants [53]. BDNF levels were significantly lower in patients with MDD than the control group, and recovery from depression after antidepressant treatment was associated with normal serum levels of BDNF in patients with MDD [54]. Similarly, there were more changes in the taxonomic composition of the microbiota in the Orange group than in the Flavored group. In the Orange group, 10 taxons, including Lachnospiraceae, Bacteroides, and Bifidobacterium, were more abundant, but only one taxon (Blautia_uc) increased in abundance in the Flavored group compared to that in the Depression_F group. Besides, the taxonomic abundance of Bifidobacterium was decreased in the Flavored group (Fig. 3F and Supplement Fig. 2B). Moreover, the relative abundance of Clostridium decreased further in the Orange group (Supplement Fig. $1 \mathrm{~F}$ and $2 \mathrm{~F}$ ). The prevalence of Clostridium was negatively associated with serum BDNF levels [38]. Therefore, we found that the 8-week intervention changed the gut microbiota composition and increased serum BDNF levels in the Orange group compared to those in the Flavored group.

Members of the Lachnospiraceae family, such as the Roseburia, Blautia, and Coprococcus genera, are known to break down carbohydrates into short-chain fatty acids (SCFAs) [55]. Reduction in these fermentation-related bacteria precipitates a decline in SCFA production, causing intestinal barrier dysfunction $[55,56]$. Moreover, 
SCFAs promote the differentiation of T cells and can function as a histone diacetylase inhibitor [57]; therefore, SCFAs can act as a regulator of immune homeostasis. Additionally, SCFAs are involved in neurotransmitter production [58] and neuroprotection and can penetrate the blood-brain barrier [59]; therefore, they have been proposed as potential novel antidepressants [60]. The Ruminococcaceae family was highly abundant in healthy controls compared to that in patients with MDD [38]. Moreover, the Ruminococcaceae family is characterized by anti-inflammatory activity and associated with a chronic low-grade inflammatory response $[49,61]$. Therefore, the relative abundance of these genera in the gut microbiome mediated the low degree of inflammation and higher intestinal barrier function. Inflammation is associated with major depression [62]. Furthermore, flavonoids exhibit a neuroprotective effect by falsifying inflammatory reactions and have potential therapeutic effects in terms of neuroprotection [63].

In this study, the use of antibiotics, probiotics, and prebiotics was not allowed for assessing the microbial community. Additionally, we served fresh, $100 \%$-pure, orange juice daily as the flavonoid-rich drink and investigated the associations between the relative abundance of the gut microbiome and depression in young adults. Although the particular association between flavonoids and depression is still unclear, we found that high intake of flavonoids changed the relative abundance of the gut microbiome and increased serum BDNF levels. An unidentified genus of Lachnospiraceae, Bacteroides, and Bifidobacterium was correlated with flavonoid-rich orange juice treatment.

\section{Conclusion}

we demonstrated that the intake of flavonoid-rich orange juice for 8 weeks has an influence on depression in young adults. Moreover, the change in gut microbiota composition due to flavonoid-rich orange juice treatment was associated with the butyrate-producing Lachnospiraceae family. Therefore, we suggest the efficacy of flavonoid-rich orange juice treatment in improving depression.

\section{Methods}

\section{Participants}

All procedures were conducted in accordance with the Declaration of Helsinki (1996) and approved by the Gachon University Institutional Review Board (IRB, 1044396-201803-HR-074-01). Forty participants (age: 20-30 years; mean age: 21.83 years with standard deviation of 2.43) were recruited from Gachon University (Gyeonggi, Korea), from April to June 2018. The participants included 16 men and 24 women. All participants were evaluated using the CES-D, which is a psychiatric screening tool to detect preexisting psychiatric disorders and has been used extensively in population-based studies [64-66]. The CES-D generated scores range from 0 to 60 , and scores $\geq 21$ are indicative of major depression. All participants of this study had a CES-D score $>21$ points [67]. Daily orange juice intake was monitored by phone and SMS, one to one by researchers. There was no dropout during the study.

All participants provided consent for participation after understanding the objective of this study, test schedule, and potential risk. Those who were using antibiotics, had a family history of mental illness, had been diagnosed with psychiatric disorders, had a history of antipsychotic medication use, or had a bowel disease were excluded from the study. 


\section{Intervention study design}

The study had a randomized, single-blind design and was conducted for 8 weeks. The volunteers were randomly divided into two groups of 20 participants each, the Orange and Flavored groups, considering the dropout rate of $20 \%$. The Flavored group consumed a placebo drink ( $190 \mathrm{~mL}$ each) and the Orange group consumed flavonoid-rich orange fresh juice (190 ml each) twice daily (30-60 min before breakfast and dinner) for 8 weeks. The participants visited the institution for screening 1 week before the beginning of the treatment and underwent the CES-D test. To check whether they met the inclusion and exclusion criteria, the registered participants returned on the first day of the treatment (1 week after the screening visit) and provided fecal specimens in a fecal collection bag or accessory bag.

FFQ and 24-h dietary recall evaluation, blood tests, and anthropometric assessments using InBody 720 (Biospace Co. Ltd., Korea) were performed. The participants also underwent the same tests at the end of the treatment (after 8 weeks).

\section{Treatment drinks}

This study used a commercially available, 100\%-pure, Florida orange juice (Natalie's Orchid Island Juice Co. \& Tomato Agricultural Association Corporation Inc., Korea) as the flavonoid-rich drink (190 mL) and a commercially available orange-flavored cordial (Del Monte Foods Inc. \& Lotte Chilsung Beverage Co. Ltd., Korea) as the LF drink $(190 \mathrm{~mL})$. The total flavonoid content was analyzed by high-performance liquid chromatography mass spectrometry. The total flavonoid content in the orange drink was $157.9 \pm 1.4 \mathrm{mg} / 100 \mathrm{~g}$ and that in the flavored drink was $28.4 \pm 0.7 \mathrm{mg} / 100 \mathrm{~g}$ (Supplement Table 4). The two drinks were almost identical in appearance, volume, taste, calories, and glucose, fructose, and sucrose levels, except in the flavonoid content (Supplement Table 5).

\section{Blood tests}

Participants were asked during screening to avoid certain foods (e.g., high-fat and high-sucrose foods, berries, fruits, fruit juice, tea, jams, and alcohol) and to have an LF diet for $24 \mathrm{~h}$ before the blood test. Fasting blood samples (5 $\mathrm{mL}$ of blood) were collected at the beginning (baseline) and at the end (8 weeks) of the treatment. Serum obtained from each blood sample was divided into $500 \mu \mathrm{L}$ aliquots and was used to measure BDNF, serotonin, folate, homocysteine, hs-CRP, and vitamin B12 levels.

\section{Dietary intake}

During the study period, the FFQ and 24-h dietary recalls were assessed twice by a qualified dietician. Using FFQ, the frequency and amount of food consumed by each participant during the past year were examined.

No separate major dietary intervention or physical activity program was offered. Moreover, participants were advised to maintain physical activity for the duration of the study.

\section{Fecal sample collection and DNA extraction}

The participants provided fecal specimens at the beginning (Depression_O, $n=20$; Depression_F, $n=20$ ) and at the end (Orange, $n=20$; Flavored, $n=20$ ) of the 8-week treatment. Fecal samples were collected using the fecal collection kit. DNA from the fecal samples was extracted using a Fast DNA ${ }^{\text {TM }}$ SPIN extraction kit (MP 
Biomedicals, Solon, Ohio, USA) according to the manufacturer's instructions. The PCR amplification and sequencing methods used in this study have been previously described [68]. Briefly, the V3 and V4 regions of the 16S rRNA gene were amplified and sequenced using Illumina MiSeq Sequencing System (Illumina, USA) by ChunLab, Inc. (Seoul, Korea).

\section{PCR amplification and Illumina sequencing}

The first PCR amplification was performed using a T100 thermal cycler (Bio-Rad, Hercules, CA, USA) to amplify the $\mathrm{V} 3$ and $\mathrm{V} 4$ regions of the $16 \mathrm{~S}$ rRNA gene. The primers used were $341 \mathrm{~F}$ ( $5^{\prime}$-TCGTCGGCAGCGTCAGATGTGTATAAGAGACAG-CCTACGGGNGGCWGCAG-3', the underlined sequence indicates the target region primer) and 805R (5'-GTCTCGTGG GCTCGG-AGATGTGTATAAGAGACAG-GACTACHVGGGTATCTAATCC-3'). The first PCR amplification was conducted under the following conditions: initial denaturation at $95^{\circ} \mathrm{C}$ for 3 min followed by 25 cycles of denaturation at $95^{\circ} \mathrm{C}$ for $30 \mathrm{~s}$, primer annealing at $55^{\circ} \mathrm{C}$ for $30 \mathrm{~s}$, and extension at $72{ }^{\circ} \mathrm{C}$ for $30 \mathrm{~s}$, with a final elongation at $72^{\circ} \mathrm{C}$ for $5 \mathrm{~min}$. The second PCR amplification to attach the lllumina NexTera barcodes was performed with the i5 forward primer (5'-AATGATACGGCGACCACCGAGATCTACACXXXXXXXX-TCGT CGGCAGCGTC-3', X indicates the barcode region) and i7 reverse primer (5'CAAGCAGAAGACGGCATACGAGAT-XXXXXXXX-AGTCTCGTGGGCTCXGG - 3'). Conditions used for the second amplification reaction were the same as those described for the first reaction except only eight amplification cycles were performed. The PCR product was confirmed using $1 \%$ agarose gel electrophoresis and visualized under a Gel Doc system (BioRad, Hercules, CA, USA). The amplified products were purified using the QIAquick PCR Purification Kit (Qiagen, Valencia, CA, USA). Equal concentrations of purified products were pooled together, and short fragments (non-target products) were removed using the Ampure beads kit (Agencourt Bioscience, MA, USA). The quality and product size were assessed on a Bioanalyzer 2100 (Agilent, Palo Alto, CA, USA) using a DNA 7500 chip. Mixed amplicons were pooled, and sequencing was performed by ChunLab, Inc. (Seoul, Korea), using the Illumina MiSeq Sequencing System (Illumina, USA) according to the manufacturer's instructions.

\section{Bioinformatics analysis}

Raw reads were checked for quality, and low-quality reads (< Q25) were filtered using Trimmomatic 0.32 [52]. After the quality control process, paired-end sequence data were merged together using PANDAseq [69]. Then, primers were trimmed using a proprietary program of ChunLab at a similarity cutoff of 0.8 . Nonspecific amplicons that did not encode 16S rRNA were detected by HMMER's hmmsearch program with 16S rRNA profiles [70]. Sequences were denoised using DUDE-Seq, and non-redundant reads were extracted by UCLUST clustering [71, 72]. The EzBioCloud database was used for taxonomic assignment using USEARCH (8.1.1861_i86linux32) followed by more precise pairwise alignment [72, 73]. UCHIME [74] and the nonchimeric $16 \mathrm{~S}$ rRNA database from EzBioCloud were used to detect chimeras on reads with a best hit similarity rate $<97 \%$. Sequence data were then clustered using CD-HIT [75] and UCLUST [72]. The alpha- and beta-diversity analyses with the Chao, Phylogenetic Diversity, Shannon, and Simpson indexes were conducted using BIOiPLUG, which is ChunLab's bioinformatics cloud platform.

\section{Preparation of genomic DNA from reference strains and fecal samples}


Fecal microbial DNA from 200 mg fecal samples was prepared using the QIAamp DNA Stool Mini Kit (Qiagen, Hilden, Germany) according to the manufacturer's instructions. DNA was quantified using a NanoDrop ND-1000 spectrophotometer (Thermo Electron) and stored at $-20^{\circ} \mathrm{C}$ before analysis.

\section{Real-time quantitative PCR}

Real-time quantitative PCR was conducted using a LightCycler 480 (Roche, Germany), and the group and species-specific primers for PCR are presented in Table 2. The primers were verified using Primer3 and BLAST and synthesized commercially. Quantitative PCR was performed in 96-well plates with final volumes of $20 \mu \mathrm{L}$, consisting of $1 \mu \mathrm{L}$ of fecal DNA, $0.5 \mu \mathrm{L}$ of primers (10 pmol each), $10 \mu \mathrm{L}$ SYBR Green I master (Roche, Mannheim, Germany), and $8 \mu \mathrm{L}$ of $\mathrm{H}_{2} \mathrm{O}$. PCR amplification involved pre-incubation at $94{ }^{\circ} \mathrm{C}$ for 4 min, followed by 55 cycles of amplification (denaturation at $94{ }^{\circ} \mathrm{C}$ for $15 \mathrm{~s}$, primer annealing at $55^{\circ} \mathrm{C}$ for $15 \mathrm{~s}$, and elongation at $72{ }^{\circ} \mathrm{C}$ for $20 \mathrm{~s}$ ). Melting curves were obtained by heating samples from $50{ }^{\circ} \mathrm{C}$ to $90{ }^{\circ} \mathrm{C}$ at a rate of $5{ }^{\circ} \mathrm{C} / \mathrm{s}$.

\section{Associations of the gut microbiota with BDNF levels and clinical indexes in patients with depression}

We assessed the associations of biomarkers and BMI with the gut microbiota in patients with depression. The correlation coefficient $(r)$ between gut microbiota and biomarkers was calculated using Spearman's rank correlation analysis. The heat map was plotted in 35 genera for participants with BDNF levels and statistically significant results $(p<0.1)$. All statistical analyses were performed using the SAS 9.4.

\section{Statistical analysis}

The Student's t-test, paired t-test, and Wilcoxon signed-rank test were performed using SPSS (version 23.0; SPSS Inc., USA). Results are expressed as mean \pm Standard Error of the Mean (SEM), and $p$ values less than 0.05 were considered to be statistically significant. $p$ values of less than $0.05,0.01$, and 0.001 are described as * $p<0.05$, ** $p<0.01$, and *** $p<0.001$, respectively.

\section{Declarations}

\section{Consent for publication}

Not applicable

\section{Availability of data and material}

Not applicable

\section{Competing interest}

All authors declare that they have no competing interest.

\section{Funding}

This work was supported by the National Research Foundation of Korea (NRF) and a grant funded by the Korean government (MSIT) (No. NRF-2016R1D1A1B03935435). 
Author Contributions

Investigation and data arrangement, M.P. and J.C. Writing, and original draft preparation, M.P. and J.C. Conceptualization and supervision, H-J.L. Funding acquisition, H-J.L.

Acknowledgements

We thank the all participants and the clinical staff that assisted with the sample collections.

\section{References}

1. (WHO) WHO: Depression and Other Common Mental Disorders- Global Health Estimates. Geneva: World Health Organization. 2017.

2. Gawlik S, Waldeier L, Müller M, Szabo A, Sohn C, Reck C: Subclinical depressive symptoms during pregnancy and birth outcome-a pilot study in a healthy German sample. Archives of Women's Mental Health 2013, 16:93-100.

3. Solem S, Hagen R, Wang CEA, Hjemdal O, Waterloo K, Eisemann M, Halvorsen M: Metacognitions and Mindful Attention Awareness in Depression: A Comparison Of Currently Depressed, Previously Depressed and Never Depressed Individuals. Clinical Psychology \& Psychotherapy 2017, 24:94-102.

4. Akbaraly TN, Brunner EJ, Ferrie JE, Marmot MG, Kivimaki M, Singh-Manoux A: Dietary pattern and depressive symptoms in middle age. The British journal of psychiatry : the journal of mental science 2009, 195:408-413.

5. Li Y, Lv M-R, Wei Y-J, Sun L, Zhang J-X, Zhang H-G, Li B: Dietary patterns and depression risk: A metaanalysis. Psychiatry Research 2017, 253:373-382.

6. Hintikka J, Tolmunen T, Honkalampi K, Haatainen K, Koivumaa-Honkanen H, Tanskanen A, Viinamäki H: Daily tea drinking is associated with a low level of depressive symptoms in the Finnish general population. European Journal of Epidemiology 2005, 20:359-363.

7. Logan AC: Omega-3 fatty acids and major depression: a primer for the mental health professional. Lipids in health and disease 2004, 3:25-25.

8. Macready AL, Kennedy OB, Ellis JA, Williams CM, Spencer JPE, Butler LT: Flavonoids and cognitive function: a review of human randomized controlled trial studies and recommendations for future studies. Genes \& nutrition 2009, 4:227-242.

9. Kaume L, Howard LR, Devareddy L: The Blackberry Fruit: A Review on Its Composition and Chemistry, Metabolism and Bioavailability, and Health Benefits. Journal of Agricultural and Food Chemistry 2012, 60:5716-5727.

10. van Praag H, Lucero MJ, Yeo GW, Stecker K, Heivand N, Zhao C, Yip E, Afanador M, Schroeter H, Hammerstone J, Gage FH: Plant-Derived Flavanol (-)Epicatechin Enhances Angiogenesis and Retention of Spatial Memory in Mice. The Journal of Neuroscience 2007, 27:5869-5878.

11. Alharbi MH, Lamport DJ, Dodd GF, Saunders C, Harkness L, Butler LT, Spencer JPE: Flavonoid-rich orange juice is associated with acute improvements in cognitive function in healthy middle-aged males. European Journal of Nutrition 2016, 55:2021-2029. 
12. Chang S-C, Cassidy A, Willett WC, Rimm EB, O'Reilly EJ, Okereke OI: Dietary flavonoid intake and risk of incident depression in midlife and older women. The American Journal of Clinical Nutrition 2016, 104:704714.

13. Mihrshahi S, Dobson AJ, Mishra GD: Fruit and vegetable consumption and prevalence and incidence of depressive symptoms in mid-age women: results from the Australian longitudinal study on women's health. European Journal Of Clinical Nutrition 2014, 69:585.

14. Pase MP, Scholey AB, Pipingas A, Kras M, Nolidin K, Gibbs A, Wesnes K, Stough C: Cocoa polyphenols enhance positive mood states but not cognitive performance: a randomized, placebo-controlled trial. Journal of Psychopharmacology 2013, 27:451-458.

15. Firoozabadi A, Kolouri S, Zarshenas MM, Salehi A, Mosavat SH, Dastgheib SA: Efficacy of Nepeta Menthoides Boiss and Buhse Freeze-Dried Aqueous Extract on Anxiety of Patients with Depression: A Double-Blind Randomized Controlled Clinical Trial. Iranian journal of medical sciences 2016, 41:S4-S4.

16. Dash S, Clarke G, Berk M, Jacka FN: The gut microbiome and diet in psychiatry: focus on depression. Current Opinion in Psychiatry 2015, 28:1-6.

17. O'Mahony SM, Clarke G, Borre YE, Dinan TG, Cryan JF: Serotonin, tryptophan metabolism and the brain-gutmicrobiome axis. Behavioural Brain Research 2015, 277:32-48.

18. Jenkins TA, Nguyen JCD, Polglaze KE, Bertrand PP: Influence of Tryptophan and Serotonin on Mood and Cognition with a Possible Role of the Gut-Brain Axis. Nutrients 2016, 8:56.

19. Desbonnet L, Garrett L, Clarke G, Bienenstock J, Dinan TG: The probiotic Bifidobacteria infantis: An assessment of potential antidepressant properties in the rat. Journal of Psychiatric Research 2008, 43:164174.

20. Desbonnet L, Garrett L, Clarke G, Kiely B, Cryan JF, Dinan TG: Effects of the probiotic Bifidobacterium infantis in the maternal separation model of depression. Neuroscience 2010, 170:1179-1188.

21. Zhang LS, Davies SS: Microbial metabolism of dietary components to bioactive metabolites: opportunities for new therapeutic interventions. Genome medicine 2016, 8:46-46.

22. Wikoff WR, Anfora AT, Liu J, Schultz PG, Lesley SA, Peters EC, Siuzdak G: Metabolomics analysis reveals large effects of gut microflora on mammalian blood metabolites. Proceedings of the National Academy of Sciences 2009, 106:3698-3703.

23. Chyan Y-J, Poeggeler B, Omar RA, Chain DG, Frangione B, Ghiso J, Pappolla MA: Potent Neuroprotective Properties against the Alzheimer $\boldsymbol{\beta}$-Amyloid by an Endogenous Melatonin-related Indole Structure, Indole-3propionic Acid. Journal of Biological Chemistry 1999, 274:21937-21942.

24. Martin-Cabezas R, Davideau J-L, Tenenbaum H, Huck O: Clinical efficacy of probiotics as an adjunctive therapy to non-surgical periodontal treatment of chronic periodontitis: a systematic review and metaanalysis. Journal of Clinical Periodontology 2016, 43:520-530.

25. Jafarnejad S, Shab-Bidar S, Speakman JR, Parastui K, Daneshi-Maskooni M, Djafarian K: Probiotics Reduce the Risk of Antibiotic-Associated Diarrhea in Adults (18-64 Years) but Not the Elderly (>65 Years). Nutrition in Clinical Practice 2016, 31:502-513.

26. Emge JR, Huynh K, Miller EN, Kaur M, Reardon C, Barrett KE, Gareau MG: Modulation of the microbiota-gutbrain axis by probiotics in a murine model of inflammatory bowel disease. American Journal of PhysiologyGastrointestinal and Liver Physiology 2016, 310:G989-G998. 
27. Dinan TG, Cryan JF: Melancholic microbes: a link between gut microbiota and depression? Neurogastroenterology \& Motility 2013, 25:713-719.

28. Huang R, Wang K, Hu J: Effect of Probiotics on Depression: A Systematic Review and Meta-Analysis of Randomized Controlled Trials. Nutrients 2016, 8:483.

29. Johnson SL, Kirk RD, DaSilva NA, Ma H, Seeram NP, Bertin MJ: Polyphenol Microbial Metabolites Exhibit Gut and Blood-Brain Barrier Permeability and Protect Murine Microglia against LPS-Induced Inflammation. Metabolites 2019, 9:78.

30. Figueira I, Garcia G, Pimpão RC, Terrasso AP, Costa I, Almeida AF, Tavares L, Pais TF, Pinto P, Ventura MR, et al: Polyphenols journey through blood-brain barrier towards neuronal protection. Scientific Reports 2017, 7:11456.

31. Fraga CG, Croft KD, Kennedy DO, Tomás-Barberán FA: The effects of polyphenols and other bioactives on human health. Food \& Function 2019, 10:514-528.

32. Lee Y, Park K: Reproducibility and validity of a semi-quantitative FFQ for trace elements. British Journal of Nutrition 2016, 116:864-873.

33. Huang Y, Shi X, Li Z, Shen Y, Shi X, Wang L, Li G, Yuan Y, Wang J, Zhang Y, et al: Possible association of Firmicutes in the gut microbiota of patients with major depressive disorder. Neuropsychiatric disease and treatment 2018, 14:3329-3337.

34. Cheung SG, Goldenthal AR, Uhlemann A-C, Mann JJ, Miller JM, Sublette ME: Systematic Review of Gut Microbiota and Major Depression. Frontiers in psychiatry 2019, 10:34-34.

35. Goodrich JK, Waters JL, Poole AC, Sutter JL, Koren O, Blekhman R, Beaumont M, Van Treuren W, Knight R, Bell JT, et al: Human genetics shape the gut microbiome. Cell 2014, 159:789-799.

36. Falony G, Joossens M, Vieira-Silva S, Wang J, Darzi Y, Faust K, Kurilshikov A, Bonder MJ, Valles-Colomer M, Vandeputte D, et al: Population-level analysis of gut microbiome variation. Science 2016, 352:560-564.

37. Naseribafrouei A, Hestad K, Avershina E, Sekelja M, Linløkken A, Wilson R, Rudi K: Correlation between the human fecal microbiota and depression. Neurogastroenterology \& Motility 2014, 26:1155-1162.

38. Jiang H, Ling Z, Zhang Y, Mao H, Ma Z, Yin Y, Wang W, Tang W, Tan Z, Shi J, et al: Altered fecal microbiota composition in patients with major depressive disorder. Brain, Behavior, and Immunity 2015, 48:186-194.

39. Lackey KA, Williams JE, Meehan CL, Zachek JA, Benda ED, Price WJ, Foster JA, Sellen DW, Kamau-Mbuthia EW, Kamundia EW, et al: What's Normal? Microbiomes in Human Milk and Infant Feces Are Related to Each Other but Vary Geographically: The INSPIRE Study. Frontiers in Nutrition 2019, 6.

40. Nuriel-Ohayon M, Neuman H, Ziv O, Belogolovski A, Barsheshet Y, Bloch N, Uzan A, Lahav R, Peretz A, Frishman S, et al: Progesterone Increases Bifidobacterium Relative Abundance during Late Pregnancy. Cell Reports 2019, 27:730-736.e733.

41. Turroni F, Taverniti V, Ruas-Madiedo P, Duranti S, Guglielmetti S, Lugli GA, Gioiosa L, Palanza P, Margolles A, van Sinderen D, Ventura M: Bifidobacterium bifidum PRL2010 Modulates the Host Innate Immune Response. Applied and Environmental Microbiology 2014, 80:730-740.

42. Kabeerdoss J, Devi RS, Mary RR, Prabhavathi D, Vidya R, Mechenro J, Mahendri NV, Pugazhendhi S, Ramakrishna BS: Effect of yoghurt containing Bifidobacterium lactis Bb12®on faecal excretion of secretory immunoglobulin A and human beta-defensin 2 in healthy adult volunteers. Nutrition Journal 2011, 10:138. 
43. Ruiz L, Delgado S, Ruas-Madiedo P, Sánchez B, Margolles A: Bifidobacteria and Their Molecular Communication with the Immune System. Frontiers in Microbiology 2017, 8.

44. Messaoudi M, Lalonde R, Violle N, Javelot H, Desor D, Nejdi A, Bisson J-F, Rougeot C, Pichelin M, Cazaubiel M, Cazaubiel J-M: Assessment of psychotropic-like properties of a probiotic formulation (Lactobacillus helveticus R0052 and Bifidobacterium longum R0175) in rats and human subjects. British Journal of Nutrition 2010, 105:755-764.

45. McGaughey KD, Yilmaz-Swenson T, Elsayed NM, Cruz DA, Rodriguiz RM, Kritzer MD, Peterchev AV, Roach J, Wetsel WC, Williamson DE: Relative abundance of Akkermansia spp. and other bacterial phylotypes correlates with anxiety- and depressive-like behavior following social defeat in mice. Scientific Reports 2019, 9:3281.

46. Tarr AJ, Galley JD, Fisher Sydney E, Chichlowski M, Berg BM, Bailey MT: The prebiotics 3'Sialyllactose and 6'Sialyllactose diminish stressor-induced anxiety-like behavior and colonic microbiota alterations: Evidence for effects on the gut-brain axis. Brain, Behavior, and Immunity 2015, 50:166-177.

47. Burokas A, Arboleya S, Moloney RD, Peterson VL, Murphy K, Clarke G, Stanton C, Dinan TG, Cryan JF: Targeting the Microbiota-Gut-Brain Axis: Prebiotics Have Anxiolytic and Antidepressant-like Effects and Reverse the Impact of Chronic Stress in Mice. Biological Psychiatry 2017, 82:472-487.

48. Belzer C, de Vos WM: Microbes inside-from diversity to function: the case of Akkermansia. The Isme Journal 2012, 6:1449.

49. Berk M, Williams LJ, Jacka FN, O’Neil A, Pasco JA, Moylan S, Allen NB, Stuart AL, Hayley AC, Byrne ML, Maes M: So depression is an inflammatory disease, but where does the inflammation come from? BMC Medicine 2013, 11:200.

50. Zheng P, Zeng B, Zhou C, Liu M, Fang Z, Xu X, Zeng L, Chen J, Fan S, Du X, et al: Gut microbiome remodeling induces depressive-like behaviors through a pathway mediated by the host's metabolism. Molecular Psychiatry 2016, 21:786.

51. Chen J-J, Zheng P, Liu Y-Y, Zhong X-G, Wang H-Y, Guo Y-J, Xie P: Sex differences in gut microbiota in patients with major depressive disorder. Neuropsychiatric disease and treatment 2018, 14:647-655.

52. Bolger AM, Lohse M, Usadel B: Trimmomatic: a flexible trimmer for Illumina sequence data. Bioinformatics 2014, 30:2114-2120.

53. Kao C-F, Liu Y-L, Yu YWY, Yang AC, Lin E, Kuo P-H, Tsai S-J: Gene-based analysis of genes related to neurotrophic pathway suggests association of BDNF and VEGFA with antidepressant treatment-response in depressed patients. Scientific Reports 2018, 8:6983.

54. Chen B, Dowlatshahi D, MacQueen GM, Wang J-F, Young LT: Increased hippocampal bdnf immunoreactivity in subjects treated with antidepressant medication. Biological Psychiatry 2001, 50:260-265.

55. Duncan SH, Louis P, Flint HJ: Cultivable bacterial diversity from the human colon. Letters in Applied Microbiology 2007, 44:343-350.

56. Vince AJ, McNeil NI, Wager JD, Wrong OM: The effect of lactulose, pectin, arabinogalactan and cellulose on the production of organic acids and metabolism of ammonia by intestinal bacteria in a faecal incubation system. British Journal of Nutrition 1990, 63:17-26.

57. Koh A, De Vadder F, Kovatcheva-Datchary P, Bäckhed F: From Dietary Fiber to Host Physiology: Short-Chain Fatty Acids as Key Bacterial Metabolites. Cell 2016, 165:1332-1345. 
58. DeCastro M, Nankova BB, Shah P, Patel P, Mally PV, Mishra R, La Gamma EF: Short chain fatty acids regulate tyrosine hydroxylase gene expression through a cAMP-dependent signaling pathway. Molecular Brain Research 2005, 142:28-38.

59. Kidd SK, Schneider JS: Protection of dopaminergic cells from MPP+-mediated toxicity by histone deacetylase inhibition. Brain Research 2010, 1354:172-178.

60. Fuchikami M, Yamamoto S, Morinobu S, Okada S, Yamawaki Y, Yamawaki S: The potential use of histone deacetylase inhibitors in the treatment of depression. Progress in Neuro-Psychopharmacology and Biological Psychiatry 2016, 64:320-324.

61. Sokol H, Pigneur B, Watterlot L, Lakhdari O, Bermúdez-Humarán LG, Gratadoux J-J, Blugeon S, Bridonneau C, Furet J-P, Corthier G, et al: <em>Faecalibacterium prausnitzii</em> is an anti-inflammatory commensal bacterium identified by gut microbiota analysis of Crohn disease patients. Proceedings of the National Academy of Sciences 2008, 105:16731-16736.

62. Lopresti AL, Maker GL, Hood SD, Drummond PD: A review of peripheral biomarkers in major depression: The potential of inflammatory and oxidative stress biomarkers. Progress in Neuro-Psychopharmacology and Biological Psychiatry 2014, 48:102-111.

63. Spagnuolo C, Moccia S, Russo GL: Anti-inflammatory effects of flavonoids in neurodegenerative disorders. European Journal of Medicinal Chemistry 2018, 153:105-115.

64. Radloff LS: The CES-D Scale:A Self-Report Depression Scale for Research in the General Population. Applied Psychological Measurement 1977, 1:385-401.

65. Bae JN, Cho MJ: Development of the Korean version of the Geriatric Depression Scale and its short form among elderly psychiatric patients. Journal of Psychosomatic Research 2004, 57:297-305.

66. Kang SY, Ah Lee J, Kim Y-S: Short report: depressive mood moderates the association between family communication and self-rated health in married couples. 2019.

67. CHO MJ, KIM KH: Use of the Center for Epidemiologic Studies Depression (CES-D) Scale in Korea. The Journal of Nervous and Mental Disease 1998, 186:304-310.

68. Jang L-G, Choi G, Kim S-W, Kim B-Y, Lee S, Park H: The combination of sport and sport-specific diet is associated with characteristics of gut microbiota: an observational study. Journal of the International Society of Sports Nutrition 2019, 16:21.

69. Masella AP, Bartram AK, Truszkowski JM, Brown DG, Neufeld JD: PANDAseq: paired-end assembler for illumina sequences. BMC Bioinformatics 2012, 13:31.

70. Eddy SR: Accelerated Profile HMM Searches. PLOS Computational Biology 2011, 7:e1002195.

71. Lee B, Moon T, Yoon S, Weissman T: DUDE-Seq: Fast, flexible, and robust denoising for targeted amplicon sequencing. PLOS ONE 2017, 12:e0181463.

72. Edgar RC: Search and clustering orders of magnitude faster than BLAST. Bioinformatics 2010, 26:24602461.

73. Myers EW, Miller W: Optimal alignments in linear space. Bioinformatics 1988, 4:11-17.

74. Edgar RC, Haas BJ, Clemente JC, Quince C, Knight R: UCHIME improves sensitivity and speed of chimera detection. Bioinformatics 2011, 27:2194-2200.

75. Fu L, Niu B, Zhu Z, Wu S, Li W: CD-HIT: accelerated for clustering the next-generation sequencing data. Bioinformatics 2012, 28:3150-3152. 


\section{Supplementary Figure Captions}

Supplementary Figure 1. Relative abundance in the Depression_0 and Orange groups.

Supplementary Figure 2. Relative abundance in the Depression_F and Flavored groups.

\section{Figures}

A
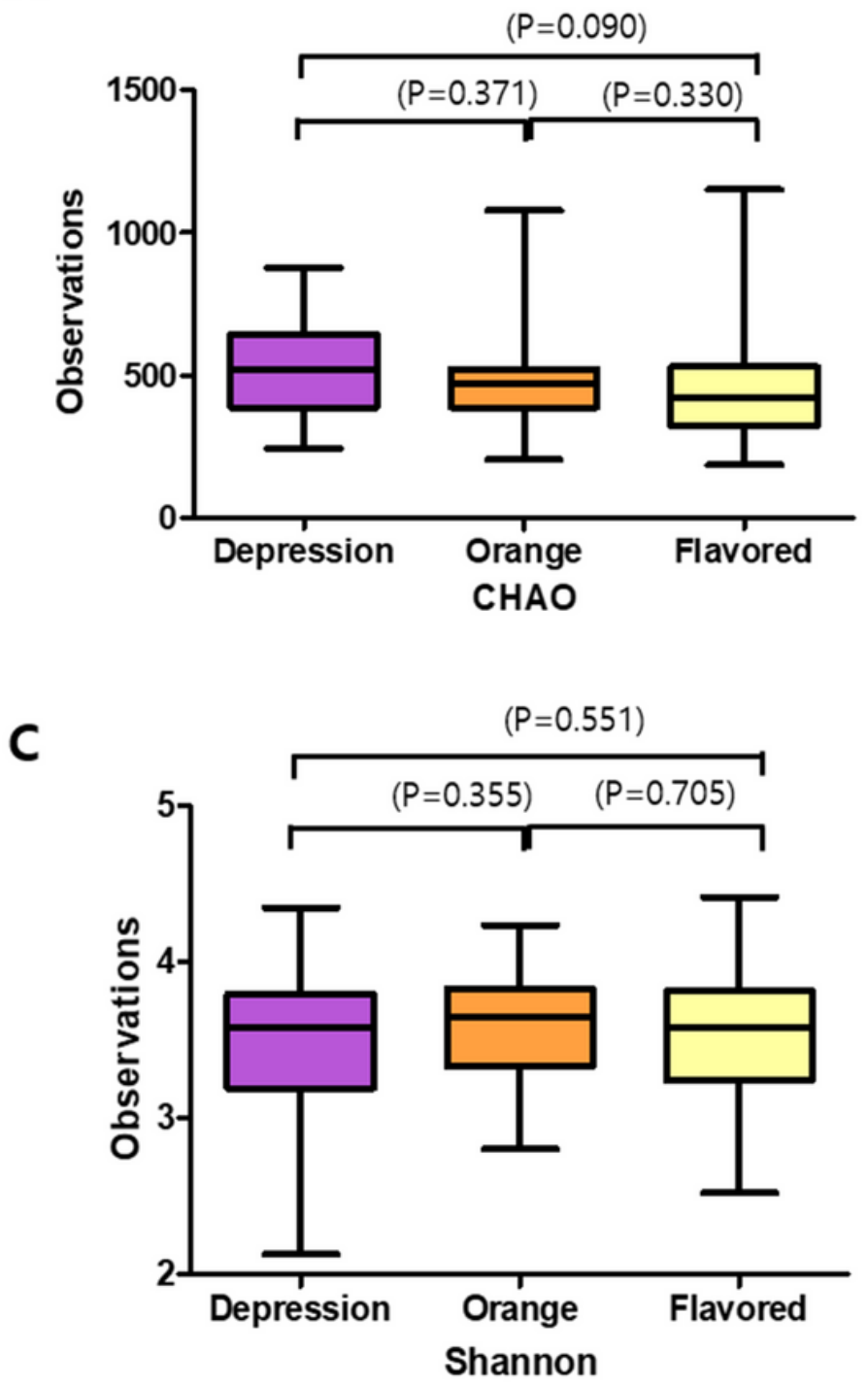

Depression
B

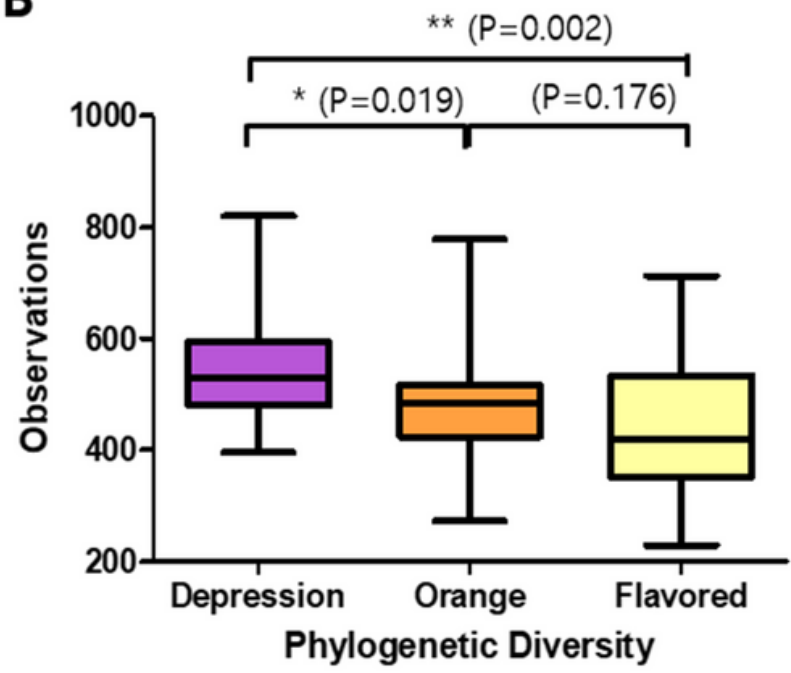

D

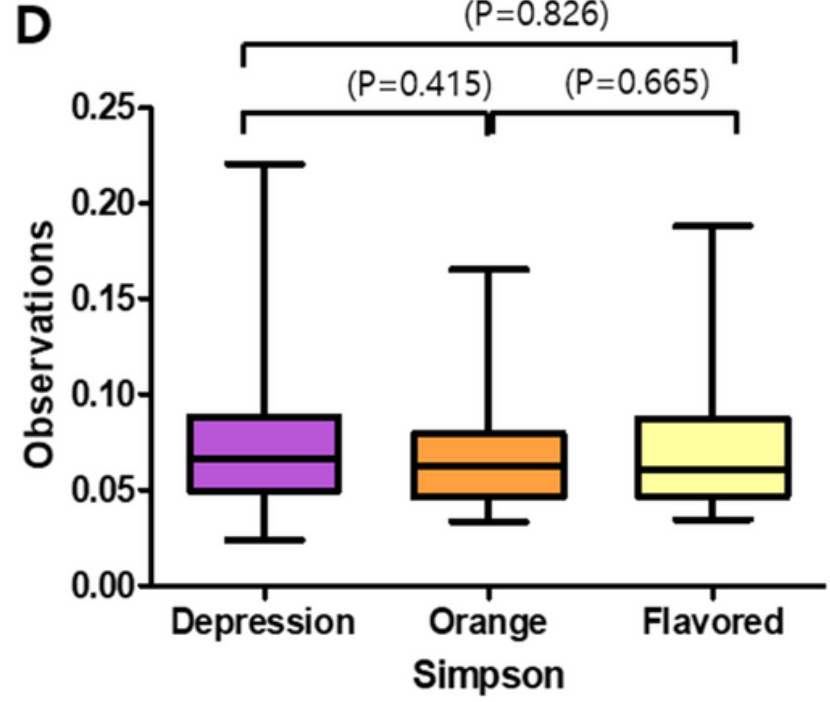

Orange $\square$ Flavored

\section{Figure 1}

Alpha-diversity index of the gut microbiota in the Depression and Orange or Flavored groups. Box plots depict greater gut microbial diversity in the Orange group than in the Depression group, according to the NP Shannon, 
Shannon, and Simpson indexes. The horizontal lines in the box plots represent median values; upper (Q3) and lower (Q1) ranges of the box represent the $75 \%$ and $25 \%$ quartiles, respectively.

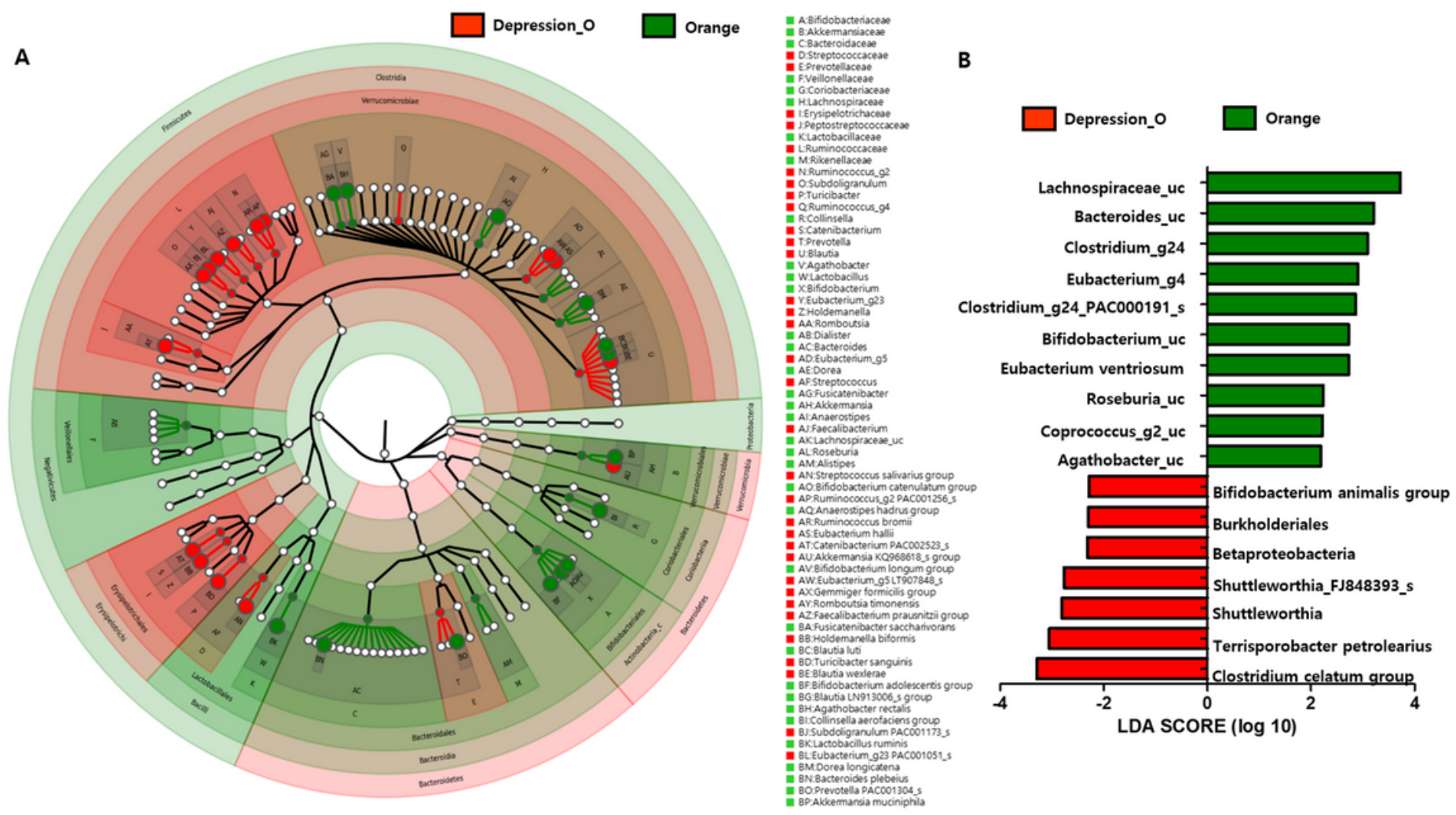

\section{Figure 2}

Alpha-diversity index of the gut microbiota in the Depression and Orange or Flavored groups. Box plots depict greater gut microbial diversity in the Orange group than in the Depression group, according to the NP Shannon, Shannon, and Simpson indexes. The horizontal lines in the box plots represent median values; upper (Q3) and lower (Q1) ranges of the box represent the $75 \%$ and $25 \%$ quartiles, respectively. 

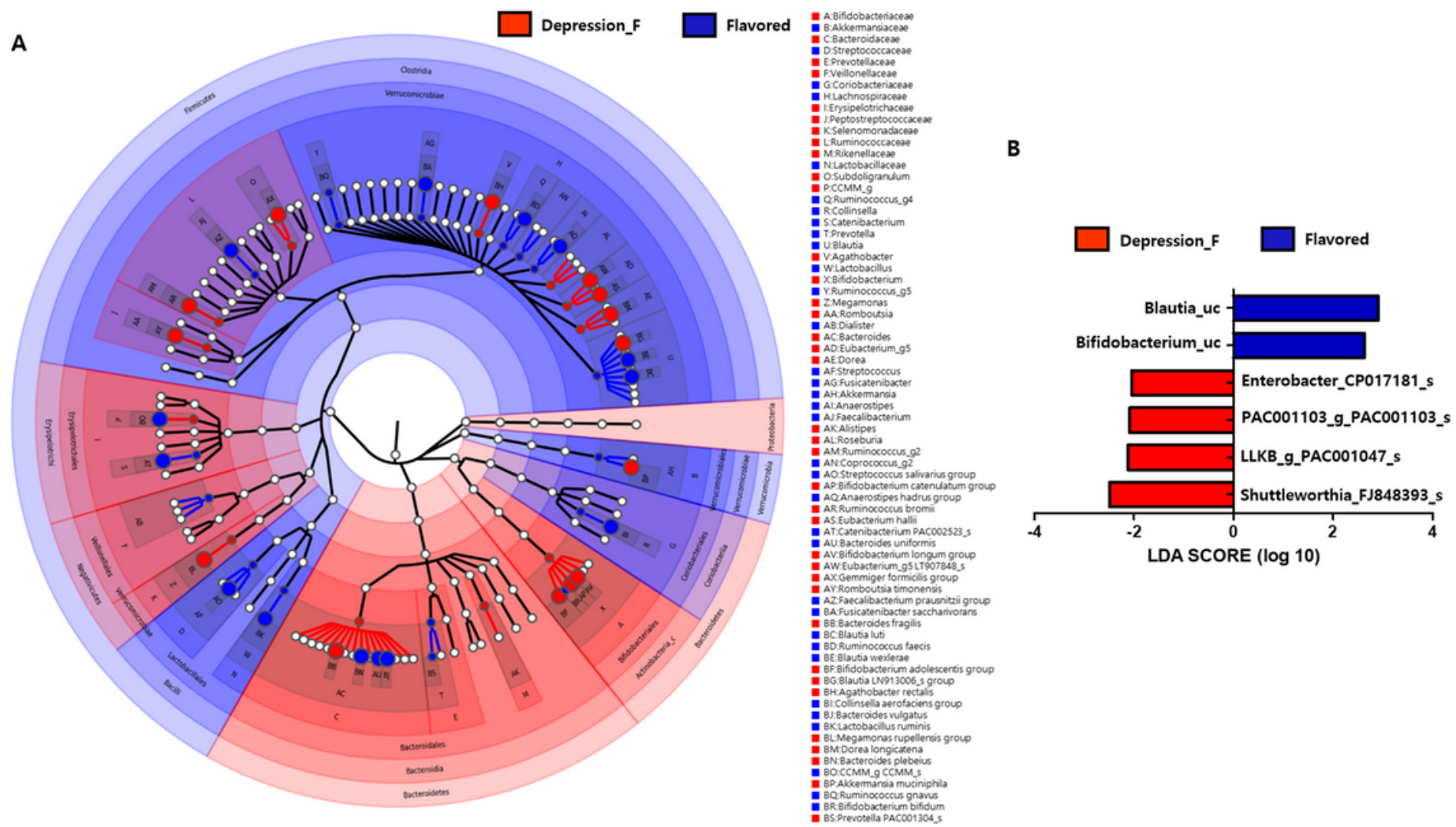

\section{Figure 3}

Genus level distribution of the gut microbiota between the Depression_F and Flavored groups. A) A cladogram of taxonomic differences between the Depression_F (red) and Flavored (blue) groups. B) Significant bacterial differences are observed in the Depression_F (red) and Flavored (blue) groups. The significant threshold of the LDA score is $>2$; flavored treatment taxonomic compositions in the Flavored group are indicated with a positive LDA (blue), and enriched taxonomic compositions in the Depression group are indicated using a negative LDA score (red). 


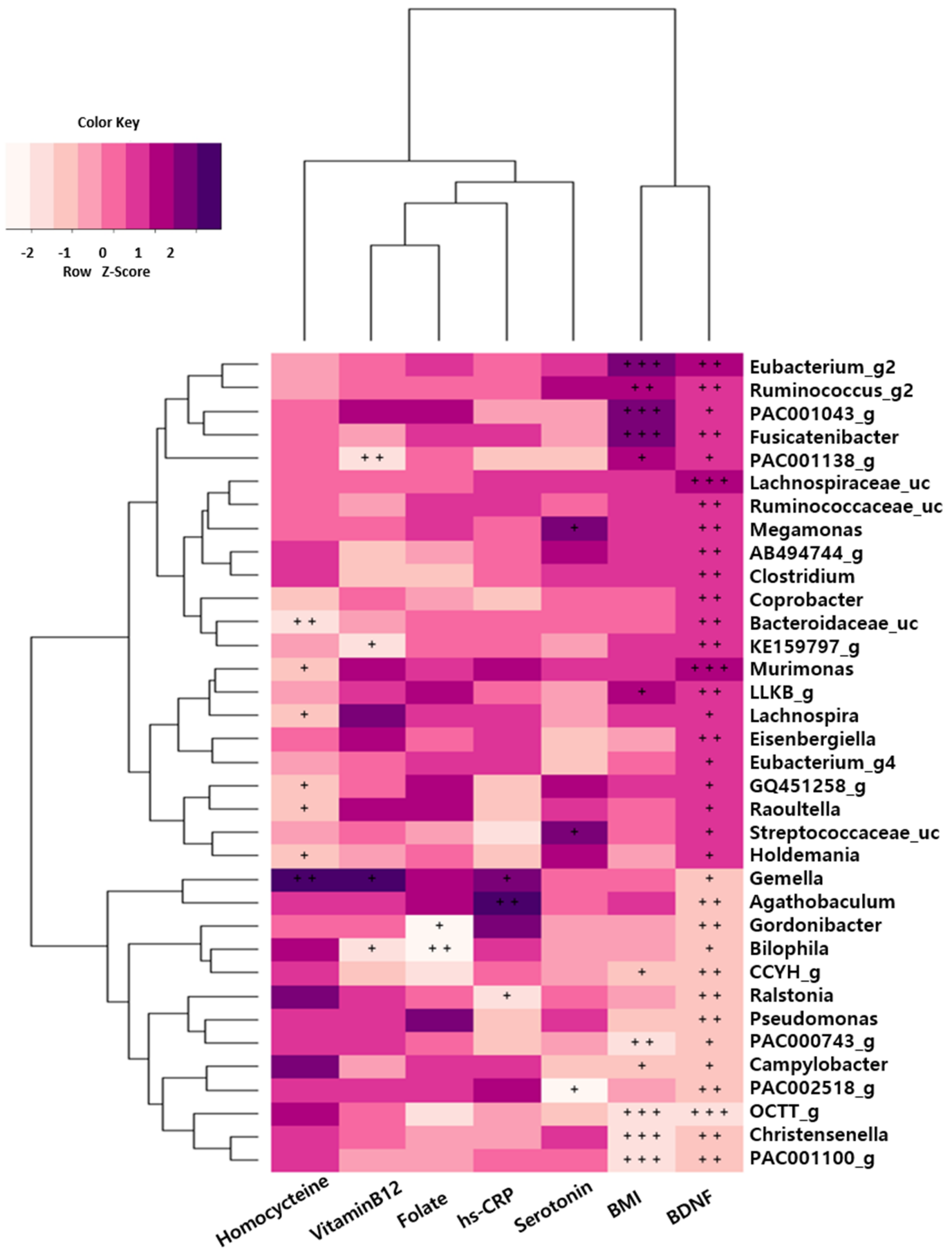

\section{Figure 4}

Associations of the gut microbiota with BDNF levels and clinical parameters in patients with depression. The heat map of Spearman's rank correlation coefficients obtained by comparing clinical parameters (BDNF scores, $p<0.05)$ and relative abundances of gut microbiota in patients with depression. The heat map was plotted using R software.,$+ \mathrm{p}<0.10 ;++, \mathrm{p}<0.05 ;+++, \mathrm{p}<0.01$. 
A

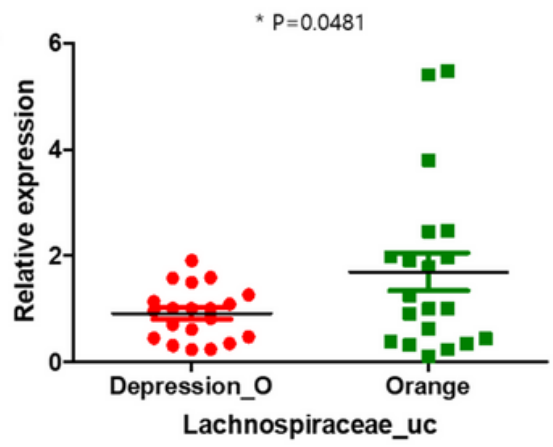

D

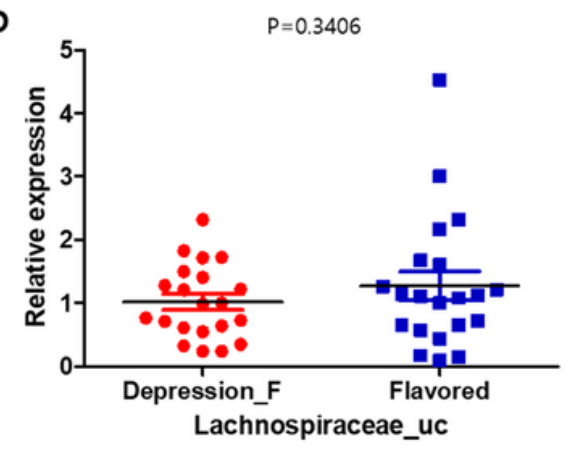

B

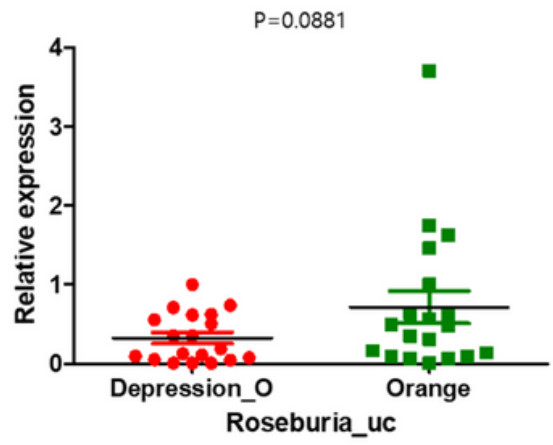

E

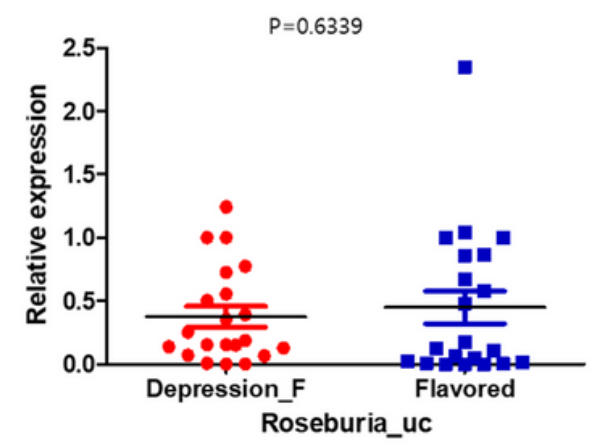

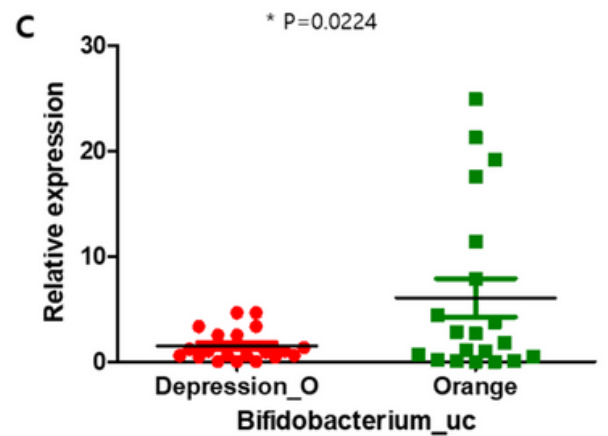

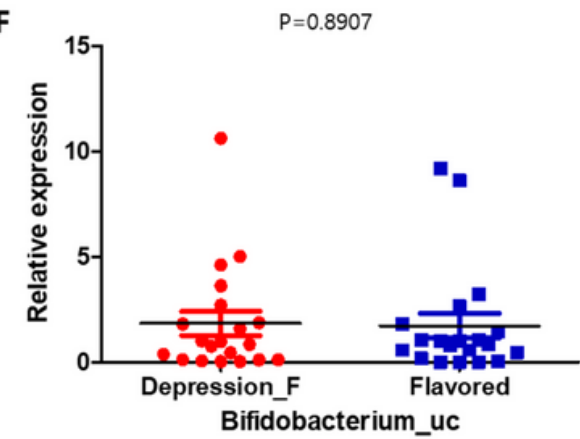

Figure 5

Quantitative changes in the gut microbiome in the Depression and Orange or Flavored groups. A) and D) unclassified Lachnospiraceae (Lachnospiraceae_uc); B) and E) unclassified Roseburia (Roseburia_uc); C) and F) unclassified Bifidobacterium (Bifidobacterium_uc) in the Orange (green) or Flavored (blue) groups. ${ }^{*} \mathrm{P}<0.05$.

\section{Supplementary Files}

This is a list of supplementary files associated with this preprint. Click to download.

- SupplementaryTables.docx

- Supplefigure2.tif

- Supplefigure1.tif 